\title{
Energy Conservation and Interference Mitigation: From Decoupling Property to Win-Win Strategy
}

\author{
Liqun Fu, Member, IEEE, Hongseok Kim, Member, IEEE, Jianwei Huang, Senior Member, IEEE, \\ Soung Chang Liew, Senior Member, IEEE, and Mung Chiang, Senior Member, IEEE
}

\begin{abstract}
This paper studies the problem of energy conservation of mobile terminals in a multi-cell TDMA network supporting real-time sessions. The corresponding optimization problem involves joint scheduling, rate control, and power control, which is often highly complex to solve. To reduce the solution complexity, we decompose the overall problem into two sub-problems: intra-cell energy optimization and inter-cell interference control. The solution of the two subproblems results in a "win-win" situation: both the energy consumptions and intercell interference are reduced simultaneously. We simulate our decomposition method with the typical parameters in WiMAX system, and the simulation results show that our decomposition method can achieve an energy reduction of more than $70 \%$ compared with the simplistic maximum transmit power policy. Furthermore, the inter-cell interference power can be reduced by more than $35 \%$ compared with the maximum transmit power policy. We find that the interference power stays largely constant throughout a TDMA frame in our decomposition method. Based on this premise, we derive an interesting decoupling property: if the idle power consumption of terminals is no less than their circuit power consumption, or when both are negligible, then the energy-optimal transmission rates of the users are independent of the inter-cell interference power.
\end{abstract}

Index Terms-Energy-efficiency, power control, rate control, scheduling, multi-cell wireless system.

\section{INTRODUCTION}

Green wireless refers to the research area that reduces the carbon footprint and energy consumption of information technology (IT) industry. There are more than 4 billion cellphones in the world [1], and wireless devices and equipments consume $9 \%$ of the total energy of IT, i.e., as much as 6.1 TWh/year [2]. Future wireless systems such as 3GPP-LTE or WiMAX2 are evolving to support broadband services that demand a higher capacity than can be provided by today's wireless network. In most cases, this is achieved at the expense of a higher energy consumption and a more severe impact on the environment. Besides the environmental concern, energy

Liqun $\mathrm{Fu}$ is with the Institute of Network Coding, The Chinese University of Hong Kong, Shatin, New Territories, Hong Kong, email: lqfu@inc.cuhk.edu.hk. Hongseok Kim is with the Department of Electronic Engineering, Sogang University, Seoul, Korea, email: hongseok@sogang.ac.kr. Jianwei Huang, and Soung Chang Liew are with the Department of Information Engineering, The Chinese University of Hong Kong, Shatin, New Territories, Hong Kong, emails:\{jwhuang,soung\}@ie.cuhk.edu.hk. Mung Chiang is with Department of Electrical Engineering, Princeton University, NJ 08544 USA, email: chiangm@princeton.edu.

This work is supported by AoE grant E-02/08 from the UGC of the Hong Kong SAR, China, the General Research Funds (Project Number 412509, 412511, 412710, and 414911) established under the University Grant Committee of the Hong Kong Special Administrative Region, China, the Sogang University Research Grant of 2011, Google, Princeton Grand Challenge grant, and NSF CNS-1011962. reduction can also lengthen the battery lifetime of terminals and improve users' experiences.

Since radio frequency $(\mathrm{RF})$ transmission consumes a significant amount of energy of terminals, we will focus on reducing their energy consumption in this paper. In particular, we will consider a time-division-multiple-access (TDMA) cellular network. In each cell, a base station serves a number of users. The transmissions of these users do not overlap in time. However, the transmissions of users in different cells may overlap and interfere with one another. Each user has a certain traffic requirement. We want to answer the following question: how do we schedule the uplink transmissions so as to minimize the total energy consumption while satisfying the traffic requirements of all users?

The gist of the problem is as follows. In the absence of interference, for a transmission, Shannon's capacity formula states that $x=w \log \left(1+\frac{p G}{\sigma^{2}}\right)$, where $x$ is the data rate, $w$ is the bandwidth, $p$ is the transmit power, $G$ is the channel gain, and $\sigma^{2}$ is the noise power. Suppose that the transmission is turned on for $T$ seconds within a frame. Then, the number of nats delivered per frame is $b=x T=w T \log \left(1+\frac{E G}{T \sigma^{2}}\right)$, where $E$ is the energy consumption per $b$ nats. From this expression, we immediately see a tradeoff between the transmission time $T$ and the energy $E$ when delivering $b$ nats: increasing the transmission time $T$ makes $E$ smaller. Multiple transmissions complicate this problem in two ways:

1) Intra-cell interaction: Each TDMA frame has a finite amount of time resource. Within each cell, a longer transmission time of one terminal means a less transmission time for other terminals. Thus, their transmit energies trade off against each other.

2) Inter-cell interaction: Across cells, the interference received by a base station depends on simultaneous transmissions in other cells. If simultaneous transmissions can be properly scheduled, mutual interferences can be reduced, which in turn can reduce the total energy consumption. This can be intuitively seen from $b=$ $w T \log \left(1+\frac{E G}{T\left(\sigma^{2}+q\right)}\right)$, where $q$ is the interference; that is, all things being equal, a smaller $E$ is required if the interference $q$ can be reduced.

Thus, to minimize the total energy consumption, we need to jointly consider the time fraction allocated to each transmission within each cell and the scheduling of simultaneously transmissions across cells. Besides the transmit energy $E$, wireless devices also consume circuit energy when they transmit, and "idle" energy when they do not. The relative 
magnitudes of these energies have a subtle but important effect on the solution to our problem.

Finding an overall optimal solution to the energy minimization problem is non-trivial, as elaborated in Section III. In this paper, we propose a method that decomposes the overall problem into two sub-problems along the line of 1 and 2 above. That is, we first consider the sub-problem of intracell time fraction allocation, assuming interference is constant throughout a frame (this assumption is to a large extent valid according to our simulation experiments - see Section V-B). After the transmission time fractions (and target SINRs) in each cell are fixed, we then consider the transmission scheduling across cells and set the transmit powers of the terminals to fulfill the target SINRs. Based on the solution to the second sub-problem, we then adjust the inter-cell interferences and solve the first sub-problem again. The process is iterated, if necessary, by alternating between these two modules.

The solution found by this decomposition method is guaranteed to be feasible, albeit not necessarily optimal. Simulations indicate that this decomposition method can achieve energy reduction of more than $70 \%$ and inter-cell interference power reduction of more than $35 \%$ compared with the simplistic scheme of maximum power transmission. We also derive an interesting decoupling property under the assumption that the inter-cell interference power stays constant over a TDMA frame: if the idle power consumption of terminals is no less than their circuit power consumption, or when both are negligible, then the energy-optimal transmission rates of the users are independent of the inter-cell interference power.

\section{A. Related Work}

Energy-efficient transmission was first explored in the context of sensor networks [3]-[6]; for example, each sensor node transmits packets as slowly as is allowed by the delay constraint, in the so-called lazy scheduling [6].

In cellular networks, most of the research on power control focuses on controlling interference, i.e., sustaining a required signal to interference-and-noise ratio (SINR) to achieve a fixed target data rate, e.g., for reliable voice connections, see [7][12] and the long list of references therein.

Recently, energy-optimal uplink scheduling for cellular systems was proposed in TDMA systems [13]. It was shown that, by properly choosing the transmit powers, as well as the instantaneous rates and the time fractions of the users within a cell, average energy consumption per real-time session can be minimized. In addition, it was demonstrated that energy saving ratio is substantial, e.g., more than $50 \%$ when the network is under-utilized. However, [13] focused on the singlecell case. The multi-cell case is of much interest because practical deployments of wireless networks contain multiple cells. Combining intra-cell time fraction allocation and intercell scheduling/power control can potentially be more energyefficient. Extensive simulations by us verify that combining energy-optimal transmission with inter-cell power control could improve the energy efficiency by $50 \%$ compared with the case when only intra-cell energy optimal transmission, as in [13], is performed. Ref. [14] studied the energy-efficient power control in OFDMA based multi-cell networks. The authors proposed a distributed non-cooperative game approach to maximize the overall network energy efficiency, which achieves a trade-off between system throughput and energy consumption.

The remainder of this paper is organized as follows. In Section II, we describe our system model and assumptions. Section III is devoted to the problem formulation. The proposed energy-efficient policy is provided in Section IV. We provide the simulation results in Section V. In Section VI, we discuss possible future works, followed by the conclusion in Section VII.

\section{SySTEM MODEL}

We consider energy efficient uplink communications in wireless cellular networks. Within each cell, the users send traffic to the same base station (BS) via Time Division Multiple Access (TDMA). The time is divided into fixed length frames. Within a frame, each user is allocated a dedicated time period, during which it is the only uplink transmitter within the cell. There is no interferences among users in the same cell. The concurrent transmissions of different users at different cells, however, lead to inter-cell interferences. We would like to choose the proper time allocations and transmission powers for users in multiple cells, such that the total energy consumption is minimized while satisfying the Quality of Service (QoS) requirements.

\section{A. Power Consumption Model}

We consider a comprehensive terminal power consumption model, which includes the transmit power, the circuit power, and the idling power [3], [13], [15], [16].

A terminal's transmission rate $x$ depends on the transmit power $p$ according to Shannon's capacity formula:

$$
x=w \log \left(1+\frac{p G}{\sigma^{2}+q}\right) \Leftrightarrow p=\left(\exp \left(\frac{x}{w}\right)-1\right) \frac{\sigma^{2}+q}{G},
$$

where $w$ is the bandwidth, $G$ is the channel gain, $\sigma^{2}$ is the noise power, and $q$ is the inter-cell interference. There is drain efficiency of the RF power amplifier at a transmitter, denoted by $\theta \in(0,1)$, which is defined as the ratio of the output power and the power consumed in the power amplifier. Therefore, given an output power of $p$, the power assumption at the RF amplifier of a transmitter is $p / \theta^{1}$.

Besides the transmit power, an active terminal also consumes non-negligible circuit power [3], [15], which is the power of the circuit blocks in the transmission chain, e.g., mixers, filters, local oscillators, and D/A converters. When a transmitter is idle, there is also power consumption due to

\footnotetext{
${ }^{1}$ In practical wireless systems, different modulation schemes and forward error correction (FEC) codes may be used. Compared with the Shannon's capacity formula, the impact of adaptive modulation and coding (AMC) schemes results in a constant SINR gap [17]. This constant factor can be absorbed by the parameter $\theta$, which denotes the cumulative effect of the drain efficiency, modulation and FEC.
} 
TABLE I

NOTATION SUMMARY

\begin{tabular}{|c|c|}
\hline Notation & Physical Meaning \\
\hline$m, n$ & the indices of cell \\
\hline$i, j$ & the indices of user \\
\hline$k$ & the index of concurrent transmission set \\
\hline $\mathcal{A}$ & the set of all users in one cell \\
\hline $\mathcal{S}$ & the concurrent transmission set \\
\hline$M$ & the number of cells \\
\hline$K$ & the number of concurrent transmission sets in one frame \\
\hline$\lambda$ & the arrival rate of users to the multi-cell network \\
\hline$r$ & $\begin{array}{ll}\text { session rate requirement } \\
\end{array}$ \\
\hline$x$ & instantaneous transmission rate \\
\hline$p$ & transmit power \\
\hline$q$ & inter-cell interference power \\
\hline$\sigma^{2}$ & noise power \\
\hline $\bar{\gamma}$ & target SINR requirement \\
\hline$w$ & spectral bandwidth \\
\hline$\alpha$ & circuit power \\
\hline$\beta$ & idling power \\
\hline$\delta$ & $\alpha-\beta$ \\
\hline$\theta$ & drain efficiency \\
\hline$G_{i(m) C(m)}$ & the channel gain of user $i(m)$ in cell $C(m)$ \\
\hline$G_{i(n) C(m)}$ & $\begin{array}{c}\text { the cross channel gain of user } i(n) \text { in cell } C(n) \text { to the } \\
\text { base station of cell } C(m)\end{array}$ \\
\hline $\bar{B}$ & $\begin{array}{ll}\text { relative channel gain matrix } \\
\end{array}$ \\
\hline$\varphi$ & Lagrange multiplier \\
\hline
\end{tabular}

leakage currents [16]. Therefore, the total power consumption $f(x)$ of a terminal with transmission rate $x$ is given as

$$
f(x)= \begin{cases}\left(\exp \left(\frac{x}{w}\right)-1\right) \frac{\sigma^{2}+q}{\theta G}+\alpha, & \text { if } x>0 \text { (active), } \\ \beta, & \text { if } x=0 \text { (idling), }\end{cases}
$$

where $\alpha$ is the circuit power when a terminal is active, and $\beta$ is the power consumed in idle state. In Section III-C and Section IV, we will show that the circuit power and the idling power have a substantial impact on the time and power solutions of energy efficient transmissions.

Main notations of this paper are summarized in Table I. We use lower boldface symbols (e.g., p) to denote vectors and uppercase boldface symbols (e.g., B) to denote matrices. We use calligraphic symbols (e.g., $\mathcal{A}$ ) to denote sets. The vector inequalities denoted by $\succeq$ and $\preceq$ are component-wise.

\section{$B$. Inter-cell Interference}

Consider a system with a set of $M$ cells: $\{C(m), 1 \leq m \leq$ $M\}$. Each cell $C(m)$ contains a set of users (terminals) $\mathcal{A}(m)$. The users within the same cell are allocated different time fractions for uplink transmissions. However, users in different cells may transmit simultaneously and cause interference to each other. As can be seen from (1), the transmit power consumption is closely related to the interference power level. Given a fixed transmission rate $x$, a larger inter-cell interference power $q$ leads to a larger transmit power $p$. Next we calculate the minimum transmit power vector and the minimum interference power vector that can support the rate requirements of several simultaneous transmissions.

Let $\mathcal{S}$ denote the set of users that are active simultaneously in the multi-cell network at a particular instant. Since TDMA is considered within each cell, the size of set $\mathcal{S}$ is no larger than the number of cells $M$, i.e., $|\mathcal{S}| \leq M$. Without loss of generality, we only need consider the $|S|$ cells with active users. Let us define an $|\mathcal{S}| \times|\mathcal{S}|$ nonnegative cross channel gain matrix $\mathbf{G}_{\mathcal{S}}=\left[g_{m n}\right]$, with entries as follows:

$$
g_{m n}= \begin{cases}0, & \text { if } m=n, \\ G_{i(n), C(m)}, & \text { if } m \neq n,\end{cases}
$$

where $G_{i(n), C(m)}$ is the channel gain from user $i(n)$ in cell $C(n)$ to the BS of cell $C(m)$. We further define an $|\mathcal{S}| \times|\mathcal{S}|$ nonnegative relative-channel-gain matrix $\mathbf{B}_{\mathcal{S}}$ of set $\mathcal{S}$, which is the cross channel gain matrix $\mathbf{G}_{\mathcal{S}}$ normalized by the direct channel gains. The elements in matrix $\mathbf{B}_{\mathcal{S}}=\left[b_{m n}\right]$ are as follows:

$$
b_{m n}= \begin{cases}0, & \text { if } m=n, \\ \frac{G_{i(n), C(m)}}{G_{i(m), C(m)}}, & \text { if } m \neq n,\end{cases}
$$

where $G_{i(m), C(m)}$ is the channel gain from user $i(m)$ in cell $C(m)$ to the BS of cell $C(m)$. Let $\gamma_{\mathcal{S}}=\left(\gamma_{i(m)}: i(m) \in \mathcal{S}\right)$ denote the target SINR vector of the users in set $\mathcal{S}$. Let $\mathbf{D}\left(\gamma_{\mathcal{S}}\right)$ be the $|\mathcal{S}| \times|\mathcal{S}|$ diagonal matrix whose diagonal entries are the elements in $\gamma_{\mathcal{S}}$. The SINR requirements of the users in set $\mathcal{S}$ can be written in matrix form as

$$
\left(\mathbf{I}-\mathbf{D}\left(\gamma_{\mathcal{S}}\right) \mathbf{B}_{\mathcal{S}}\right) \mathbf{p}_{\mathcal{S}} \succeq \mathbf{D}\left(\gamma_{\mathcal{S}}\right) \mathbf{v}_{\mathcal{S}}
$$

where $\mathbf{I}$ is an $|\mathcal{S}| \times|\mathcal{S}|$ identity matrix, and vector $\mathbf{v}_{\mathcal{S}}=$ $\left(\frac{\sigma^{2}}{G_{i(m), C(m)}}: i(m) \in \mathcal{S}\right)^{T}$ is the noise power vector normalized by the channel gain.

Let $\rho\left(\mathbf{D}\left(\gamma_{\mathcal{S}}\right) \mathbf{B}_{\mathcal{S}}\right)$ denote the largest real eigenvalue (also called the Perron-Frobenius eigenvalue or the spectral radius) of matrix $\mathbf{D}\left(\gamma_{\mathcal{S}}\right) \mathbf{B}_{\mathcal{S}}$. The following well-known proposition gives the necessary and sufficient condition of checking the feasibility of a target SINR vector $\gamma_{\mathcal{S}}$ and computing the minimum transmit power solutions that achieves $\gamma_{\mathcal{S}}$.

Proposition 1 ([18]-[20]): The necessary and sufficient condition for a target SINR vector $\gamma_{\mathcal{S}}$ to be feasible is

$$
\rho\left(\mathbf{D}\left(\gamma_{\mathcal{S}}\right) \mathbf{B}_{\mathcal{S}}\right)<1
$$

If $\gamma_{\mathcal{S}}$ is feasible, the component-wise minimum transmit power to achieve $\gamma_{\mathcal{S}}$ is

$$
\mathbf{p}_{\mathcal{S}}\left(\gamma_{\mathcal{S}}\right)=\left(\mathbf{I}-\mathbf{D}\left(\gamma_{\mathcal{S}}\right) \mathbf{B}_{\mathcal{S}}\right)^{-1} \mathbf{D}\left(\gamma_{\mathcal{S}}\right) \mathbf{v}_{\mathcal{S}}
$$

Proof sketch: By the Perron-Frobenius theorem [18], we know that $\rho\left(\mathbf{D}\left(\gamma_{\mathcal{S}}\right) \mathbf{B}_{\mathcal{S}}\right)$ is a positive, simple eigenvalue of matrix $\mathbf{D}\left(\gamma_{\mathcal{S}}\right) \mathbf{B}_{\mathcal{S}}$, and its corresponding eigenvector is positive componentwise. From matrix theory, we know that $\rho\left(\mathbf{D}\left(\gamma_{\mathcal{S}}\right) \mathbf{B}_{\mathcal{S}}\right)<1$ is a necessary and sufficient condition for $\left(\mathbf{I}-\mathbf{D}\left(\gamma_{\mathcal{S}}\right) \mathbf{B}_{\mathcal{S}}\right)^{-1}$ to exist [19]. Furthermore, [20] shows that (7) is a Pareto-optimal solution to (5). That is, any transmit power $\mathbf{p}$ that satisfies (5) is component-wise no smaller than $\mathbf{p}_{\mathcal{S}}\left(\gamma_{\mathcal{S}}\right)$, i.e., $\mathbf{p} \succeq \mathbf{p}_{\mathcal{S}}\left(\gamma_{\mathcal{S}}\right)$.

The total interference and noise power at the BS of cell $C(m)$ is given by

$$
q_{C(m)}=\sum_{i(n) \in \mathcal{S}, n \neq m} G_{i(n), C(m)} \cdot p_{i(n)}+\sigma^{2},
$$


which can be written in matrix form as

$$
\mathbf{q}=\mathbf{G}_{\mathcal{S}} \cdot \mathbf{p}+\boldsymbol{\eta}_{\mathcal{S}} .
$$

Proposition 2 ( [9]): The interference power vector of set $\mathcal{S}$ corresponding to the minimum transmit power solution in (7) is given by

$$
\mathbf{q}_{\mathcal{S}}\left(\gamma_{\mathcal{S}}\right)=\left(\mathbf{I}-\mathbf{B}_{\mathcal{S}} \mathbf{D}\left(\gamma_{\mathcal{S}}\right)\right)^{-1} \boldsymbol{\eta}_{\mathcal{S}}
$$

where $\boldsymbol{\eta}_{\mathcal{S}}=\left(\sigma^{2}, \sigma^{2}, \cdots, \sigma^{2}\right)^{T}$ is the noise power vector. Each element in $\mathbf{q}_{\mathcal{S}}\left(\gamma_{\mathcal{S}}\right)$ denotes the interference power received by the corresponding base station. Furthermore, $\mathbf{q}_{\mathcal{S}}\left(\gamma_{\mathcal{S}}\right)$ is the component-wise minimum interference power vector with the target SINR vector $\gamma_{\mathcal{S}}$. That is, for any transmit power solution $\mathbf{p}$ that achieves an SINR vector no less than $\gamma_{\mathcal{S}}$, its corresponding interference power vector $\mathbf{q}$ satisfies

$$
\mathbf{q} \succeq \mathbf{q}_{\mathcal{S}}\left(\gamma_{\mathcal{S}}\right)
$$

Proof: The interference power vector corresponding to the transmit power solution $\mathbf{p}_{\mathcal{S}}\left(\gamma_{\mathcal{S}}\right)$ in $(7)$ is

$$
\begin{aligned}
\mathbf{q}_{\mathcal{S}}\left(\gamma_{\mathcal{S}}\right) & =\mathbf{G}_{\mathcal{S}} \cdot \mathbf{p}_{\mathcal{S}}\left(\gamma_{\mathcal{S}}\right)+\boldsymbol{\eta}_{\mathcal{S}} \\
& =\mathbf{G}_{\mathcal{S}}\left(\mathbf{I}-\mathbf{D}\left(\gamma_{\mathcal{S}}\right) \mathbf{B}_{\mathcal{S}}\right)^{-1} \mathbf{D}\left(\gamma_{\mathcal{S}}\right) \mathbf{v}_{\mathcal{S}}+\boldsymbol{\eta}_{\mathcal{S}} \\
& =\mathbf{B}_{\mathcal{S}}\left(\mathbf{I}-\mathbf{D}\left(\gamma_{\mathcal{S}}\right) \mathbf{B}_{\mathcal{S}}\right)^{-1} \mathbf{D}\left(\gamma_{\mathcal{S}}\right) \boldsymbol{\eta}_{\mathcal{S}}+\boldsymbol{\eta}_{\mathcal{S}} \\
& =\left(\mathbf{B}_{\mathcal{S}}\left(\mathbf{I}-\mathbf{D}\left(\gamma_{\mathcal{S}}\right) \mathbf{B}_{\mathcal{S}}\right)^{-1} \mathbf{D}\left(\gamma_{\mathcal{S}}\right)+\mathbf{I}\right) \boldsymbol{\eta}_{\mathcal{S}} \\
& =\left(\mathbf{I}-\mathbf{B}_{\mathcal{S}} \mathbf{D}\left(\gamma_{\mathcal{S}}\right)\right)^{-1} \boldsymbol{\eta}_{\mathcal{S}} .
\end{aligned}
$$

For any transmit power solution $\mathbf{p}$ that achieves an SINR vector no less than $\gamma_{\mathcal{S}}$, we have $\mathbf{p} \succeq \mathbf{p}_{\mathcal{S}}\left(\gamma_{\mathcal{S}}\right)$. Furthermore the cross channel gain matrix $\mathbf{G}_{\mathcal{S}}$ is non-negative. According to (8), the interference power vector corresponding to $\mathbf{p}$ satisfies $\mathbf{q} \succeq \mathbf{q}_{\mathcal{S}}\left(\gamma_{\mathcal{S}}\right)$.

\section{Dynamic User Sessions}

We study a dynamic system with real-time application sessions (e.g., video/voice sessions). Our target is to minimize the average energy consumption per session in a stationary system. We assume that the users' arrival to each cell $C(m)$ follows a Poisson process with rate $\lambda_{C(m)}$. Then the arrival rate to all the cells is $\lambda=\sum_{m=1}^{M} \lambda_{C(m)}$. Let $J$ be a random variable denoting the energy consumption per session and $P$ be a random variable denoting the total power consumption in the system. The following proposition shows the relation between $\mathrm{E}[P]$ and $\mathrm{E}[J]$ in a stationary system:

Proposition 3 ( [13]): In a stationary system with user arrival rate $\lambda$, we have $\mathrm{E}[P]=\lambda \mathrm{E}[J]$.

According to Proposition 3, minimizing the average energy consumption per session is equivalent to minimizing the average power consumption of all the users in the system. Furthermore, there is a special feature for real-time sessions: the connection duration of a real-time session is independent of the allocated transmission rate. For example, allocating a higher transmission rate to a voice session cannot make the phone call end earlier, and the stationary distribution of the number of users in the TDMA system is independent of the transmit powers as long as the rate requirements are satisfied
[13]. Therefore, minimizing the energy consumption in a dynamic system that supports real-time sessions is equivalent to minimizing the energy consumption with a static number of users in the TDMA system ${ }^{2}$. In the rest of the paper, we will focus on the average power minimization problem in the multi-cell system with a static number of users.

\section{Problem Formulation AND Decoupling}

\section{A. Power Minimization in Multi-Cell Networks}

We assume that the frames are synchronized across all cells in the multi-cell network. Without loss of generality, the frame duration is normalized to be 1 . Since different users are active at different times in different cells, we will have different concurrent transmission sets in the multi-cell network. Suppose there are a total $K$ concurrent transmission sets, denoted by $\left\{\mathcal{S}_{k}, 1 \leq k \leq K\right\}$. Each set $\mathcal{S}_{k}$ is active for a time fraction of $t_{k}\left(0 \leq t_{k} \leq 1\right)$ within a frame. If we consider all possible combinations of simultaneous active users, then $K$ can be as large as $\prod_{m=1}^{M}(|\mathcal{A}(m)|+1)$. For example, in a multicell network with 19 cells with each cell having 9 users, we have $K=10^{19}$. Let $\mathbf{x}_{\mathcal{S}_{k}}=\left(x_{i(m)}(k): i(m) \in \mathcal{S}_{k}\right)$ denote the instantaneous transmission rate vector of set $\mathcal{S}_{k}$. According to Shannon's capacity formula, the relation between the instantaneous transmission rate vector $\mathbf{x}_{\mathcal{S}_{k}}$ and the corresponding SINR vector $\gamma_{\mathcal{S}_{k}}$ is

$$
\mathbf{x}_{\mathcal{S}_{k}}=w \log \left(1+\gamma_{\mathcal{S}_{k}}\right) \Leftrightarrow \gamma_{\mathcal{S}_{k}}=\exp \left(\frac{\mathbf{x}_{\mathcal{S}_{k}}}{w}\right)-1 .
$$

Substituting (10) into (7), then the minimal power vector $\mathbf{p}_{\mathcal{S}_{k}}$ that supports $\mathbf{x}_{\mathcal{S}_{k}}$ is

$$
\begin{aligned}
\mathbf{p}_{\mathcal{S}_{k}}\left(\mathbf{x}_{\mathcal{S}_{k}}\right)=\left(\mathbf{I}-\mathbf{D}\left(\exp \left(\frac{\mathbf{x}_{\mathcal{S}_{k}}}{w}\right)-1\right) \mathbf{B}_{\mathcal{S}}\right)^{-1} . \\
\mathbf{D}\left(\exp \left(\frac{\mathbf{x}_{\mathcal{S}_{k}}}{w}\right)-1\right) \mathbf{v}_{\mathcal{S}} .
\end{aligned}
$$

Recall that $\mathcal{A}(m)$ is the set of users in cell $C(m)$. For a user $i(m) \in \mathcal{A}(m)$ with real-time sessions, its QoS requirement is measured as its session rate requirement $r_{i(m)}$. We assume that there is call admission control that guarantees that the system load is no larger than the system capacity. This guarantees that the rate requirements of all the users admitted to system can be satisfied. As shown in Section II-C, under Proposition 3 , given an arrival rate $\lambda$ to the system, the average energy consumption per session is proportional to the expected power usage of all users at a moment in time in a stationary system. Thus minimizing the average energy per session is equivalent to minimizing the expected power usage of the system in a multi-cell system. To represent this problem mathematically, we define the following binary coefficients for each user $i(m) \in \mathcal{A}(m), 1 \leq m \leq M$, and $1 \leq k \leq K$,

$$
z_{i(m)}(k)= \begin{cases}1, & \text { if } i(m) \in \mathcal{S}_{k}, \\ 0, & \text { if } i(m) \notin \mathcal{S}_{k} .\end{cases}
$$

\footnotetext{
${ }^{2}$ This only holds for dynamic systems that support real-time sessions, but does not hold for other non-real-time sessions such as file transfer. For delaytolerant non-real-time sessions, the stationary distribution of the number of users heavily depends on the rate and power control allocations of the users. For example, allocating a lower transmission rate to a file transfer session will keep the corresponding user staying longer in the system.
} 
Problem: Average Power Minimization in a Multi-cell Network

$$
\begin{aligned}
\operatorname{minimize} & \sum_{k=1}^{K} t_{k}\left(\sum _ { m = 1 } ^ { M } \left(\sum _ { i ( m ) \in \mathcal { A } ( m ) } \left(\left(1-z_{i(m)}(k)\right) \beta\right.\right.\right. \\
& \left.\left.\left.+z_{i(m)}(k)\left(\alpha+\frac{p_{i(m)}(k)}{\theta}\right)\right)\right)\right)
\end{aligned}
$$

subject to $\sum_{k=1}^{K} t_{k}=1$,

$$
\sum_{k=1}^{K} z_{i(m)}(k) \cdot x_{i(m)}(k) \cdot t_{k}=r_{i(m)}, \forall i(m), \forall m,
$$

variables $x_{i(m)}(k) \geq 0, \quad \forall k, \forall i(m), \forall m$,

$$
t_{k} \geq 0, \quad \forall k \text {. }
$$

The objective function in (13) is the total average power consumption of all the users in the system and consists of two parts. The first part is the power consumption when the users are idle. The second part is the power consumption when the users are active in transmissions, where $p_{i(m)}(k)$ is computed according to (11) as a function of $\mathbf{x}_{\mathcal{S}_{k}}$. The first constraint in (13) states that the total time allocated to all the concurrent transmission sets equals the frame length, which is normalized to be 1 . Here, we treat the case where no user is active in any cell as a special concurrent transmission set of $\mathcal{S}_{k}=\emptyset$. The second constraint in (13) states that each user's session rate requirement is satisfied. The variables in (13) are the time fraction variables $t_{k}$ and the instantaneous rate variables $x_{i(m)}(k)$.

It is challenging to solve Problem (13) directly and optimally. First, if we consider all possible combinations of simultaneous active users, then the total number of concurrent transmission sets $K$ increases exponentially with the cell number $M$. Second, the transmit power $p_{i(m)}(k)$ in the objective function of (13) is a complicated function of the instantaneous rate variables $x_{i(m)}(k)$ 's. The transmit power is different for each user $i(m)$ and each different concurrent transmission set $\mathcal{S}_{k}$.

In this paper, we focus on designing a heuristic algorithm to solve Problem (13) based on one key assumption:

Assumption 1: For each cell $C(m)$, we assume the interference experienced by the BS, $q(m)$, remains constant within a time frame.

Assumption 1 is later verified reasonable with the simulation results in Section V-B. With this assumption, the users' transmission schedule in one cell does not affect the transmissions in other cells. Without loss of generality, we will simply assume that the transmission order of the users in each cell is fixed based on the arrival order of the corresponding sessions. We will tackle Problem (13) by solving intra-cell average power minimization and inter-cell power control separately.

\section{B. Intra-Cell Average Power Minimization}

Based on Assumption 1, the average power minimization problem of a given cell turns out to be a convex optimization problem. Let us consider cell $C(m)$. The session rate requirement of user $i(m) \in \mathcal{A}_{m}$ is $r_{i(m)}$. If the instantaneous transmission rate of $i(m)$ is $x_{i(m)}$, then the time fraction that user $i(m)$ needs to satisfy its session rate requirement is $t_{i(m)}=$ $\frac{r_{i(m)}}{x_{i(m)}}$. During the time fraction $t_{i(m)}$, the power consumption of the active user $i(m)$ is $\frac{\exp \left(\frac{x_{i(m)}}{w}\right)-1}{\theta G_{i(m) C(m)}}\left(\sigma^{2}+q(m)\right)+\alpha$. All other users in cell $C(m)$ remain in idle state during $t_{i(m)}$. The power consumption of all idle users during the time fraction $t_{i(m)}$ is $(|\mathcal{A}(m)|-1) \beta$. If $1-\sum_{i \in \mathcal{A}(m)} \frac{r_{i(m)}}{x_{i(m)}}>0$, then all user$\mathrm{s}$ will remain idle during the time fraction of $1-\sum_{i \in \mathcal{A}(m)} \frac{r_{i(m)}}{x_{i(m)}}$, with the total power consumption of $|\mathcal{A}(m)| \beta$. The intra-cell average power minimization problem can be formulated as follows:

\section{Problem: Intra-Cell Average Power Minimization:}

$$
\begin{array}{ll}
\text { minimize } & \sum_{i(m) \in \mathcal{A}(m)} \frac{r_{i(m)}}{x_{i(m)}}\left(\frac{\exp \left(\frac{x_{i(m)}}{w}\right)-1}{\theta G_{i(m) C(m)}}\right. \\
& \left.\left(\sigma^{2}+q(m)\right)+\alpha+(|\mathcal{A}(m)|-1) \beta\right) \\
& +\left(1-\sum_{i \in \mathcal{A}(m)} \frac{r_{i(m)}}{x_{i(m)}}\right)|\mathcal{A}(m)| \beta \\
\text { subject to } & \sum_{i(m) \in \mathcal{A}(m)} \frac{r_{i(m)}}{x_{i(m)}} \leq 1 \\
\text { variables } & x_{i(m)} \geq 0, \forall i(m) \in \mathcal{A}(m) .
\end{array}
$$

The objective in (14) is to minimize the total average power consumptions of all users in cell $C(m)$ during the unit time frame. Since we consider uplink transmissions, the base station is the common receiver for all the users in $\mathcal{A}(m)$. Thus, the inter-cell interference power at the base station (i.e., $q(m)$ ) is the same for every user. The constraint in (14) states that the total active time fraction is no larger than the frame length.

Problem (14) can be shown to be equivalent to,

$$
\begin{aligned}
& \text { minimize } \sum_{i(m) \in \mathcal{A}(m)} \frac{r_{i(m)}}{x_{i(m)}}\left(\frac{\exp \left(\frac{x_{i(m)}}{w}\right)-1}{\theta G_{i(m) C(m)}}\right. \\
& \left.\left(\sigma^{2}+q(m)\right)+\alpha-\beta\right) \\
& \text { subject to } \sum_{i(m) \in \mathcal{A}(m)} \frac{r_{i(m)}}{x_{i(m)}} \leq 1 \\
& \text { variables } \quad x_{i(m)} \geq 0, \forall i(m) \in \mathcal{A}(m) \text {. }
\end{aligned}
$$

If we change the variable $x_{i(m)}$ to the time fraction variable 
$t_{i(m)}=\frac{r_{i(m)}}{x_{i(m)}}$, Problem (15) is further equivalent to,

$$
\begin{array}{ll}
\text { minimize } & \sum_{i(m) \in \mathcal{A}(m)} t_{i(m)}\left(\frac{\exp \left(\frac{r_{i(m)}}{w t_{i(m)}}\right)-1}{\theta G_{i(m) C(m)}}\right. \\
& \left.\left(\sigma^{2}+q(m)\right)+\alpha-\beta\right) \\
\text { subject to } & \sum_{i(m) \in \mathcal{A}(m)} t_{i(m)} \leq 1 \\
\text { variables } & t_{i(m)} \geq 0, \forall i(m) \in \mathcal{A}(m) .
\end{array}
$$

The second derivative of the objective function in (16) with respect to variable $t_{i(m)}$ is

$$
\frac{\left(\sigma^{2}+q(m)\right) r_{i(m)}^{2}}{\theta G_{i(m) C(m)} w^{2} t_{i(m)}^{3}} \exp \left(\frac{r_{i(m)}}{w t_{i(m)}}\right),
$$

which is always positive. So the objective function in (16) is convex. The constraints in (16) are linear constraints. Therefore, Problem (16) is a convex optimization problem. The optimal instantaneous rate $x_{i(m)}^{*}$ (or equivalently the optimal time fraction $t_{i(m)}^{*}$ ) of the intra-cell power minimization problem in general depends on the inter-cell interference power $q(m)$. To simplify notation, let $\delta=\alpha-\beta$.

Next we show that the optimal solutions to the intra-cell power minimization problem and the inter-cell interference power can be decoupled if $\delta \leq 0$.

\section{Decoupling Property When $\delta \leq 0$}

If $\delta \leq 0$, the idling power $\beta$ is no smaller than the circuit power $\alpha$. Then we have the following theorem. In addition, the theorem is also valid when both the circuit power and the idling power are negligible (i.e., $\beta \approx \alpha \approx 0$ ).

Theorem 1: If $\delta \leq 0$, the optimal instantaneous transmission rate solutions, the optimal time fractions, and the optimal target SINRs of the intra-cell power minimization problem (15) (i.e., $x_{i(m)}^{*}, t_{i(m)}^{*}$, and $\gamma_{i(m)}^{*}$ for all $i(m) \in \mathcal{A}(m)$ ) are independent of the inter-cell interference power level, the circuit power, and the idling power.

Proof: The first order derivative of the objective function in (16) with respect to variable $t_{i(m)}$ is

$$
\begin{aligned}
\frac{\sigma^{2}+q(m)}{\theta G_{i(m) C(m)}}\left(-\frac{r_{i(m)}}{w t_{i(m)}} \exp \left(\frac{r_{i(m)}}{w t_{i(m)}}\right)+\right. & \\
& \left.\exp \left(\frac{r_{i(m)}}{w t_{i(m)}}\right)-1\right)+\delta .
\end{aligned}
$$

The first part of (17) (except $\delta$ ) is always negative when $0 \leq t_{i(m)} \leq 1$. This can be easily shown if we let $u_{i(m)}=$ $\frac{r_{i(m)}}{w t_{i(m)}}$. The first part of (17) then becomes

$$
\frac{\sigma^{2}+q(m)}{\theta G_{i(m) C(m)}}\left(-u_{i(m)} \exp \left(u_{i(m)}\right)+\exp \left(u_{i(m)}\right)-1\right) \text {. }
$$

The first order derivative of (18) with respect to $u_{i(m)}$ is $\frac{\sigma^{2}+q(m)}{\theta G_{i(m) C(m)}}\left(-u_{i(m)} \exp \left(u_{i(m)}\right)\right)$, which is negative for any positive $u_{i(m)}$. So (18) is a monotonically decreasing function of $u_{i(m)}$. When $u_{i(m)}=0$, (18) equals zero. So (18) is negative for any positive $u_{i(m)}$. When $0 \leq t_{i(m)} \leq 1$, we have $u_{i(m)} \geq \frac{r_{i(m)}}{w}$. So the first part of (17) is always negative when $0 \leq t_{i(m)} \leq 1$.

Therefore, when $\delta \leq 0$, (17) is always negative. So the object function in (16) is a monotonically decreasing function of the transmission time fraction $t_{i(m)}$. As a result, the optimal solution to Problem (16) is achieved when the inequality constraint is tight, i.e., $\sum_{i(m) \in \mathcal{A}(m)} t_{i(m)}=1$. In this case, minimizing

$$
\sum_{i(m) \in \mathcal{A}(m)} t_{i(m)}\left(\frac{\exp \left(\frac{r_{i(m)}}{w \cdot t_{i(m)}}\right)-1}{\theta G_{i(m) C(m)}}\left(\sigma^{2}+q(m)\right)+\delta\right)
$$

is equivalent to minimizing

$$
\sum_{i(m) \in \mathcal{A}(m)} t_{i(m)}\left(\frac{\exp \left(\frac{r_{i(m)}}{w \cdot t_{i(m)}}\right)-1}{\theta G_{i(m) C(m)}}\left(\sigma^{2}+q(m)\right)\right) .
$$

Furthermore, $\sigma^{2}+q(m)$ becomes a common scaling factor in the objective function and thus can be removed. Therefore, Problem (16) is equivalent to a simplified formulation where $q(m)$ and $\delta$ can be removed:

$$
\begin{array}{ll}
\text { minimize } & \sum_{i(m) \in \mathcal{A}(m)} t_{i(m)}\left(\frac{\exp \left(\frac{r_{i(m)}}{w \cdot t_{i(m)}}\right)-1}{G_{i(m) C(m)}}\right) \\
\text { subject to } & \sum_{i(m) \in \mathcal{A}(m)} t_{i(m)}=1 \\
\text { variables } & t_{i(m)} \geq 0 .
\end{array}
$$

This completes the proof.

The physical meaning of Theorem 1 is that if $\delta \leq 0$ (i.e., the idle power consumption is no less than the circuit power consumption), the users in the system will make use of all the time resource for transmissions in order to minimize the system power consumption. When the whole time frame is utilized, the interference power at the base station is a common influence that affects all the users in the cell, which does not affect the time fraction allocation among the users in the system. Theorem 1 will be referred to the "decoupling property" for $\delta \leq 0$, which decouples the intra-cell average power optimization from the inter-cell power control.

\section{ThE DSP ALGORITHM}

Theorem 1 motivates us to propose an algorithm, called Decomposed Scheduling and Power control (DSP), to achieve energy-efficient transmissions in a multi-cell system. Different values of $\delta$ will lead to different executions in the algorithm.

\section{A. DSP Algorithm When $\delta \leq 0$}

Because of the decoupling property when $\delta \leq 0$, we will optimize the average power consumption in two separate steps:

- Step 1 (intra-cell average power minimization): Each cell $C(m)$ solves Problem (19) to determine the optimal time fraction, the optimal instantaneous rate, and the optimal target SINR of each user in $\mathcal{A}(m)$. 
- Step 2 (inter-cell power control): Given the optimal target SINRs of the users in each cell, we can get the optimal target SINR vector for the users that are active simultaneously (i.e., in each set $\mathcal{S}_{k}$ ). Then we will compute the component-wise minimum power solution that satisfies the target SINR vector.

The flowchart of the DSP algorithm for the case $\delta \leq 0$ is shown in Fig. 1.

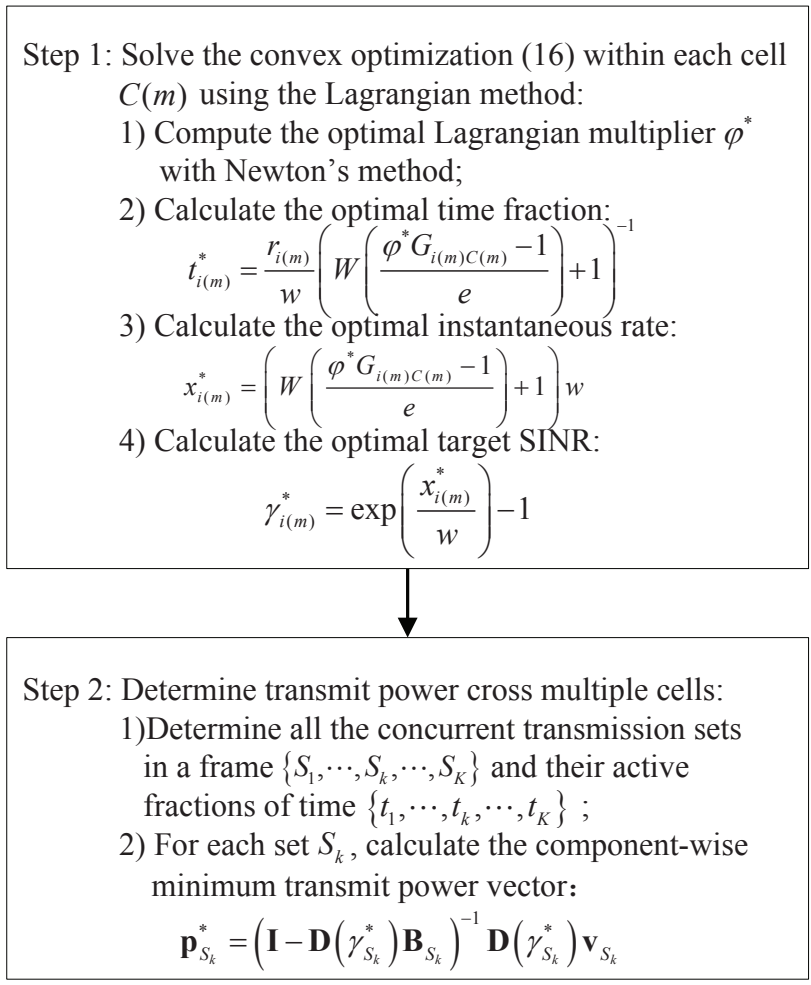

Fig. 1. Flowchart of the DSP method for the case $\delta \leq 0$

In Step 1, each cell $C(m)$ solves the convex optimization problem (19) using the Lagrangian method. Let $\varphi$ denote the Lagrangian multiplier of the constraint in (19). The Lagrangian function is

$$
\begin{aligned}
L(\mathbf{t}, \varphi)=\sum_{i(m) \in \mathcal{A}(m)} t_{i(m)} & \left(\frac{\exp \left(\frac{r_{i(m)}}{w \cdot t_{i(m)}}\right)-1}{G_{i(m) C(m)}}\right) \\
& +\varphi\left(\sum_{i(m) \in \mathcal{A}(m)} t_{i(m)}-1\right) .
\end{aligned}
$$

Since Problem (19) is convex, the necessary and sufficient conditions for an optimal solution are the KKT conditions:

$$
\nabla_{\mathbf{t}} L(\mathbf{t}, \varphi)=0 \quad \text { and } \quad \varphi\left(\sum_{i(m) \in \mathcal{A}(m)} t_{i(m)}-1\right)=0
$$

From $\nabla_{\mathbf{t}} L(\mathbf{t}, \varphi)=0$, we have

$$
\varphi^{*}=\frac{1}{G_{i(m) C(m)}}\left(\exp \left(\frac{r_{i(m)}}{w t_{i(m)}^{*}}\right)\left(\frac{r_{i(m)}}{w t_{i(m)}^{*}}-1\right)+1\right),
$$

where $\varphi^{*}$ is the optimal Lagrange multiplier and $t_{i(m)}^{*}$ is the optimal time fraction solution to (19). Given the parameters of $r_{i(m)}, G_{i(m) C(m)}$, and $w$, the optimal Lagrange multiplier $\varphi^{*}$ can be computed by the Newton's method, which guarantees superlinear convergence (faster than exponential) [21]. After obtaining $\varphi^{*}$, the optimal time fraction $t_{i(m)}^{*}$ can be calculated by solving (20). An efficient way to solve (20) is to tabulate the Lambert $W$ function [22], which is defined as

$$
W(y) \exp (W(y))=y .
$$

Then $t_{i(m)}^{*}$ is given by

$$
t_{i(m)}^{*}=\frac{r_{i(m)}}{w}\left(W\left(\frac{\varphi^{*} G_{i(m) C(m)}-1}{e}\right)+1\right)^{-1} .
$$

The optimal instantaneous rate solution $x_{i(m)}^{*}$ is:

$$
x_{i(m)}^{*}=\frac{r_{i(m)}}{t_{i(m)}^{*}}=\left(W\left(\frac{\varphi^{*} G_{i(m) C(m)}-1}{e}\right)+1\right) w .
$$

Given the instantaneous rate solution $x_{i(m)}^{*}$, the target SINR $\gamma_{i(m)}^{*}$ then can be determined by equation (10).

In Step 2, optimal power control is performed across multiple cells to determine the optimal transmit powers for the users in each cell. We have obtained the active time fraction $t_{i(m)}^{*}$, the instantaneous rate $x_{i(m)}^{*}$, and the target SINR $\gamma_{i(m)}^{*}$ of each user in each cell. Because the scheduling order in each cell is determined by its arrival order, we can determine all the concurrent transmission sets $\left\{\mathcal{S}_{k}, 1 \leq k \leq K\right\}$ and their active fractions of time $\left\{t_{k}, 1 \leq k \leq K\right\}$ in the frame. According to Proposition 1, we can compute the component-wise minimum transmit power solutions of each set $\mathcal{S}_{k}$ that achieve the target SINR vector $\gamma_{\mathcal{S}_{k}}^{*}$ as in (7).

\section{B. DSP Algorithm When $\delta>0$}

When $\delta>0$, the circuit power is greater than the idling power, which is more likely to happen in practice [3]. The intra-cell power minimization problem for $\delta>0$ is given in (16). The optimal time fraction and the optimal instantaneous rate solution to (16) are dependent on the inter-cell interference power $q(m)$. This motivates us to use an iterative method to minimize the energy consumption in the multi-cell network. At the beginning of each iteration, we replace $q(m)$ with the average interference power $\hat{q}(m)$ obtained from the previous iteration for every cell $C(m)$. For the first iteration, the estimated interference power $\hat{q}(m)$ is the averaged interference power of the previous frame.

The flowchart of the DSP algorithm for the case of $\delta>$ 0 is shown in Fig. 2. It involves an iteration between two steps. In Step 1, each cell $C(m)$ solves Problem (16) using the Lagrangian method, where $q(m)$ is replaced by $\hat{q}(m)$. The 


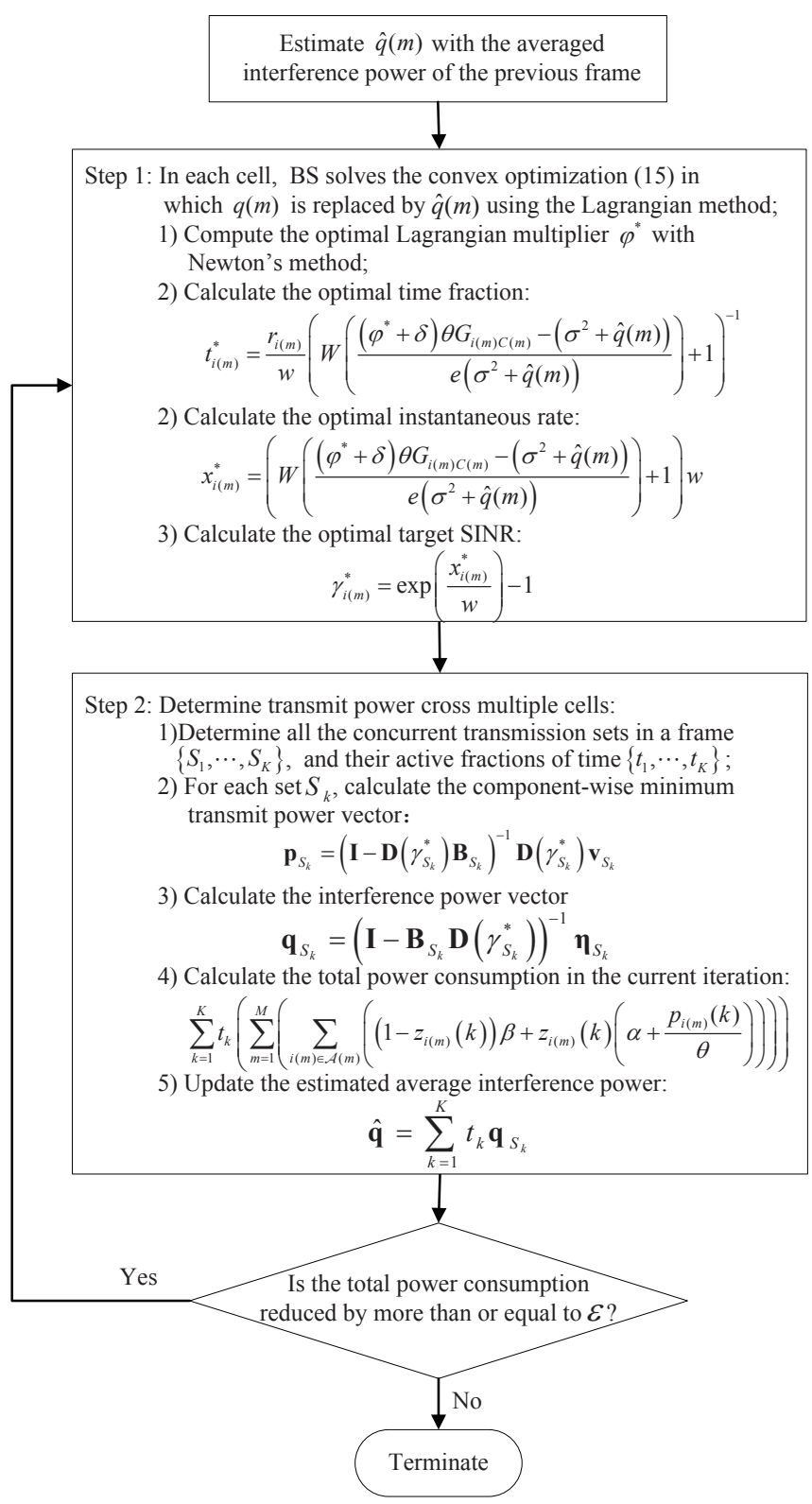

Fig. 2. Flowchart of the DSP method when $\delta>0$

Lagrangian function of (16) is given by

$$
\begin{aligned}
& L(\mathbf{t}, \varphi)=\sum_{i(m) \in \mathcal{A}(m)} t_{i(m)}\left(\frac{\exp \left(\frac{r_{i(m)}}{w \cdot t_{i(m)}}\right)-1}{\theta G_{i(m) C(m)}}\right. \\
&\left.\left(\sigma^{2}+\hat{q}(m)\right)+\delta\right)+\varphi\left(\sum_{i(m) \in \mathcal{A}(m)} t_{i(m)}-1\right) .
\end{aligned}
$$

Similarly, we use the KKT conditions to solve formulation (16). Compared with (20), (21), and (22), the optimal Lagrange multiplier $\varphi^{*}$, the optimal time fraction $t_{i(m)}^{*}$, and the optimal instantaneous rate $x_{i(m)}^{*}$ under the case of $\delta>0$ are modified to

$$
\begin{gathered}
\varphi^{*}=\frac{\sigma^{2}+\hat{q}(m)}{\theta G_{i(m) C(m)}}\left(\exp \left(\frac{r_{i(m)}}{w t_{i(m)}^{*}}\right)\left(\frac{r_{i(m)}}{w t_{i(m)}^{*}}-1\right)+1\right)-\delta, \\
t_{i(m)}^{*}=\frac{r_{i(m)}}{w} . \\
\left(W\left(\frac{\left(\varphi^{*}+\delta\right) \theta G_{i(m) C(m)}-\left(\sigma^{2}+\hat{q}(m)\right)}{e\left(\sigma^{2}+\hat{q}(m)\right)}\right)+1\right)^{-1},
\end{gathered}
$$

and

$$
\begin{aligned}
& x_{i(m)}^{*}=\frac{r_{i(m)}}{t_{i(m)}^{*}}= \\
& \quad\left(W\left(\frac{\left(\varphi^{*}+\delta\right) \theta G_{i(m) C(m)}-\left(\sigma^{2}+\hat{q}(m)\right)}{e\left(\sigma^{2}+\hat{q}(m)\right)}\right)+1\right) w .
\end{aligned}
$$

In Step 2, given the active time fraction $t_{i(m)}^{*}$, the instantaneous rate $x_{i(m)}^{*}$, and the target SINR $\gamma_{i(m)}^{*}$ obtained in step 1 , the concurrent transmission sets $\left\{\mathcal{S}_{k}, 1 \leq k \leq K\right\}$ and their active fractions of time $\left\{t_{k}, 1 \leq k \leq K\right\}$ are determined. The transmit power vector $\mathbf{p}_{\mathcal{S}_{k}}$ and the interference power vector $\mathrm{q}_{\mathcal{S}_{k}}$ for each set $\mathcal{S}_{k}$ can be determined according to equations (7) and (9), respectively. The total power consumption in the current iteration is computed by

$$
\begin{array}{r}
\sum_{k=1}^{K} t_{k}\left(\sum _ { m = 1 } ^ { M } \left(\sum _ { i ( m ) \in \mathcal { A } ( m ) } \left(\left(1-z_{i(m)}(k)\right) \beta+z_{i(m)}(k)\right.\right.\right. \\
\left.\left.\left.\left(\alpha+\frac{p_{i(m)}(k)}{\theta}\right)\right)\right)\right),
\end{array}
$$

where $z_{i(m)}(k)$ (defined in (12)) denotes whether user $i(m)$ is active in set $\mathcal{S}_{k}$, and $p_{i(m)}(k)$ is the $m$ th element in the transmit power vector $\mathbf{p}_{\mathcal{S}_{k}}$.

We use the averaged interference power vector in the current frame to serve as the estimate interference power in the next iteration, which is given by

$$
\hat{\mathbf{q}}=\sum_{k=1}^{K} t_{k} \mathbf{q}_{\mathcal{S}_{k}}
$$

The $m$ th element in vector $\hat{\mathbf{q}}$ is the averaged interference power experienced by the BS in cell $C(m), \hat{q}(m)$. Notice that in each iteration of the DSP algorithm, the total power consumption is compared with last iteration, and the next iteration starts if the total power consumption is reduced by more than or equal to a percentage threshold $\varepsilon \in(0,1)$. If the improvement of the total power consumptions is less than $\varepsilon$, the DSP algorithm terminates. The total power consumption is monotonically decreasing and the DSP algorithm is guaranteed to converge in a finite number of iterations ${ }^{3}$.

${ }^{3}$ The maximum number of iterations is upper bounded by $\log _{\varepsilon}\left(\frac{P_{\min }}{P_{1}}\right)$, where $P_{1}$ is the total power consumption in the first iteration and $P_{\min }$ is the minimum total power consumption in the system. 


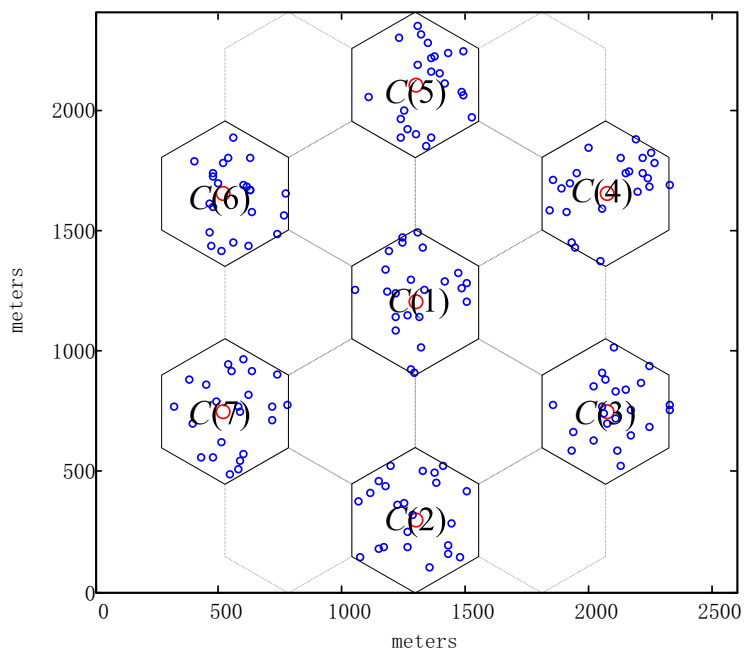

Fig. 3. A multi-cell network with 7 cells operated on the same channel (the frequency reuse factor is 3 ), and there are 23 users uniformly distributed in each cell. The red circles are the base stations and the small blue circles are the users. Here we only show the users which transmit on one particular channel.

\section{Simulation Results}

We carry out extensive simulations to evaluate the performance of the proposed DSP algorithm. We simulate a multicell network with a frequency reuse factor of 3 , i.e., one of every 3 cells use the same channel. The network topology is shown in Fig. 3. There are a total of 7 cells using the same channel, and the radius of each cell is $300 \mathrm{~m}$. The users are uniformly distributed in each cell. For a given number of users, we investigate 100 sets of random user positions and present the averaged results. The session rate requirement of each user is $70 \mathrm{kbps}$ (48.52 knats/second). The bandwidth is $1 \mathrm{MHz}$. The frame length is normalized to be 1 second. The maximum output power is $27.5 \mathrm{dBm}$. The drain efficiency is 0.2 . The noise power density is $-174 \mathrm{dBm} / \mathrm{Hz}$. The power related parameters are cited from [13], [16]. We adopt the distance-based path loss model with a path loss exponent of 4.

\section{A. Power Consumption Improvement}

We evaluate the performance of the DSP algorithm proposed for both the two cases where $\delta \leq 0$ and $\delta>0$. For $\delta \leq 0$, we only consider the transmit power consumption and neglect the circuit power and the idling power consumption. Then the algorithm in Section IV-A is used. For $\delta>0$, the idling power and the circuit power are set as $25 \mathrm{~mW}$ and $30 \mathrm{~mW}$, respectively, and therefore the algorithm in Section IV-B is used. The improvement threshold $\varepsilon$ is set as $0.001 \%$.

We compare the power consumption performances of the following three transmission policies:

1) Maximum power transmission: each user transmits with the same maximum transmit power.

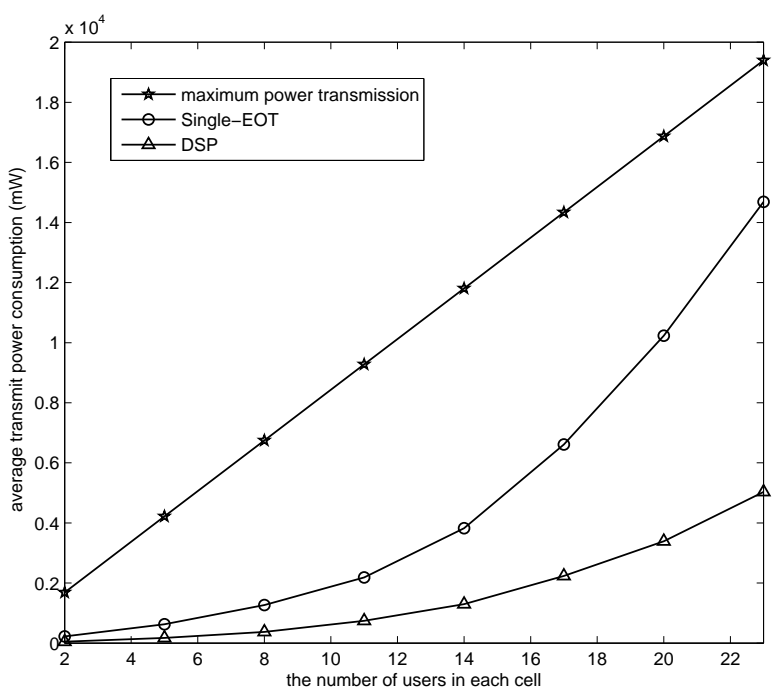

Fig. 4. Transmit power consumptions, $\delta=0$ and the algorithm in Section IV-A is used. The number of users in each cell ranges from 2 to 23 .

2) Single-EOT: the Single-cell Energy Optimal Transmission policy proposed in [13] ${ }^{4}$.

3) DSP: Decomposed Scheduling and Power control proposed in this paper.

Figure 4 shows the system power consumptions of the above three algorithms as a function of the number of users in each cell when only the transmit power consumption is considered. Figure 5 shows the system total power consumptions including the transmit power, the circuit power, and the idling power. As expected, DSP outperforms single-EOT, which in turn outperforms the maximum transmit power policy in both Fig. 4 and Fig. 5. The system power consumptions of the SingleEOT and DSP algorithms increase more slowly as the number of users increases. Because the connection duration of a real time session is the same among these three algorithms, so the system power reduction ratio is equivalent to the system energy reduction ratio. For all simulation settings (i.e., the number of users per cell ranges from 2 to 23), compared with the maximum transmit power policy, DSP achieves a power/energy reduction of more than $74 \%$ and $70 \%$ in Fig. 4 and Fig. 5, respectively. The energy saving benefits become more significant when only the transmit power consumption is considered.

In single-EOT, the BS trades off energy consumption and transmission time from a single cell's perspective. However, since BSs of different cells do not cooperate in singleEOT, the power saving is still limited due to conservative estimation of the inter-cell interferences. The DSP algorithm

\footnotetext{
${ }^{4}$ Reference [13] considered an isolated single cell network, where the intercell interference power is 0 . Here we consider multi-cell network extension. In order to make sure the target transmission rate can be achieved when the actual interference power is unknown, we assume the worst case inter-cell interference power. In this case, the BS assumes that the users in the adjacent cells use maximum transmit power, and the worst case interference distance is twice of the cell radius.
} 


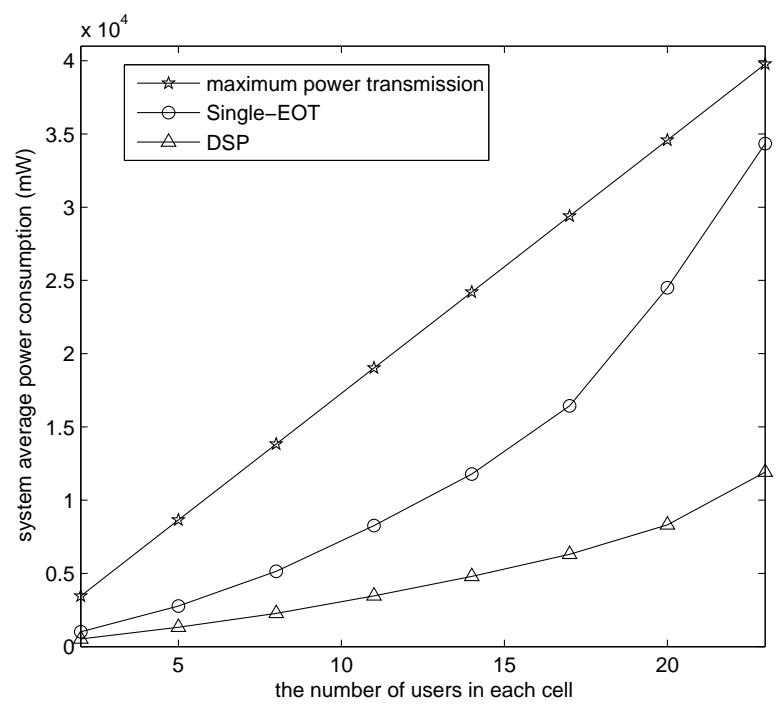

Fig. 5. System total power consumptions, $\delta>0$ and the algorithm in Section IV-B is used. The number of users in each cell ranges from 2 to 23 .

combines the intra-cell average power minimization with intercell power control. As a result, the system power/energy consumption reduction ratio can be further improved compared with the Single-EOT algorithm: for all the simulated numbers of users per cell, DSP algorithm achieves a further system power/energy reduction of more than $65 \%$ and $50 \%$ in Fig. 4 and Fig. 5, respectively.

\section{B. Inter-cell Interference Level}

We next investigate the interference power levels of the DSP algorithm when $\delta>0$. Specifically, we focus on the interference power at the base station of the central cell $C(1)$ in the network topology in Fig. 3.

Figure 6 shows the average interference power as a function of the number of users in each cell. It is clear that DSP outperforms single-EOT, which in turn outperforms the maximum transmit power policy. The maximum transmit power policy not only consumes a large system power consumption but also generates a large interference power at the base station. Compared with the maximum transmit power policy, DSP achieves an interference power reduction of more than $35 \%$ for all the simulated number of users per cell. DSP leads to a "win-win" situation: it reduces both the transmit power and the inter-cell interference. Furthermore, we find that there is a tradeoff between the interference power levels and the system work load in both the Single-EOT and DSP algorithms. The interference power levels of the Single-EOT and DSP increase as the number of users increases. When each cell has a small number of users, each user has more time to transmit and thus the inter-cell interference powers can be reduced significantly. However, in the maximum transmit power policy, the interference power levels are similar as the number of users changes.

We further investigate how the interference power changes over time. Figure 7 exhibits the interference power levels

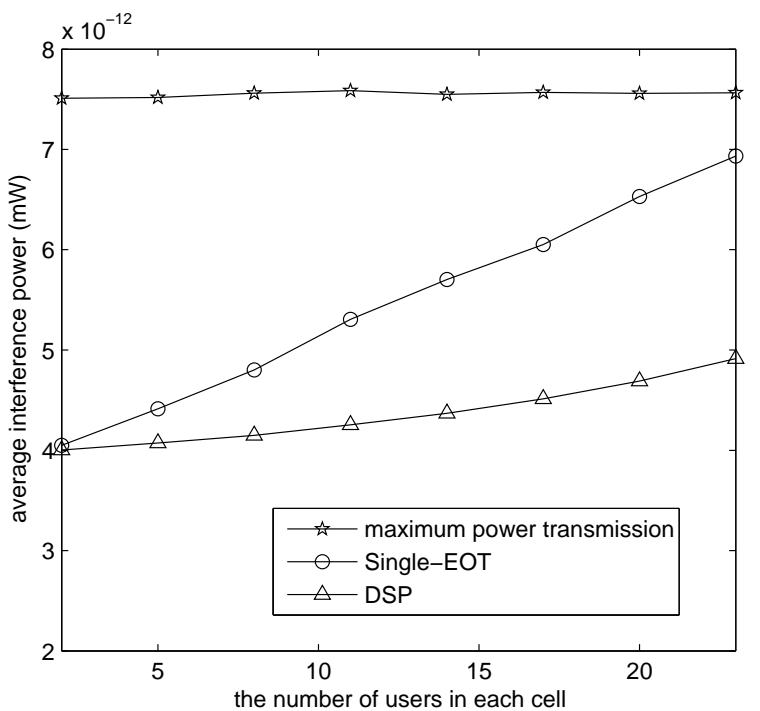

Fig. 6. Averaged interference power at the base station of the center cell $C(1)$, where the number of users in each cell ranges from 2 to 23 .

of a sample network with 23 users uniformly distributed in each cell under the maximum transmit power policy and the DSP algorithm. The $\mathrm{x}$-axis represents the time within a single frame. The $y$-axis is the interference power at the base station of cell $C(1)$. Figure 7 shows that the interference power at the base station fluctuates a lot in the maximum transmit power policy; however the interference power remains roughly constant within a time frame in the DSP algorithm.

Specifically, to measure the fluctuation of the interference power, we examine the coefficient of variation. Given the interference power vector that contains all the interference powers at the base station of $C(1)$ within a time duration of one frame, the coefficient of variation is defined by the ratio between the standard deviation and the mean of the interference. A large coefficient of variation indicates a large fluctuation of the interference power within the frame. Quantitatively, the coefficient of variation of the interference power in Fig. 7 under the maximum transmit power policy is 0.1316 . Under the DSP algorithm, the coefficient of variation is reduced to 0.0146 . This is because under the maximum transmit power policy, each user in the adjacent cells $(C(2)$ to $C(7))$ uses the same transmit power. The interference power at base station of $C(1)$ heavily depends on the locations of the active users in cells $C(2)$ to $C(7)$. If a user is at the cell boundary that is close to the base station of $C(1)$, it will generate a large interference. In the DSP algorithm, after doing single-cell optimization, the user at the cell boundary is allocated a larger fraction of time resource so that its instantaneous transmission rate requirement can be reduced. Therefore, the transmit power of the cell-boundary user can be reduced, which causes less interference to the base station of cell $C(1)$.

Table II shows the averaged coefficient of variation when the number of users in each cell changes ranges from 2 to 23 . We find that for all the simulated numbers of users per cell, the averaged coefficients of variation of the DSP algorithm is 
TABLE II

The Averaged Coefficient of Variation of the Interference Power at the Base Station of Cell $C(1)$

\begin{tabular}{c|c|c|c|c|c|c|c|c}
\hline & $n=2$ & $n=5$ & $n=8$ & $n=11$ & $n=14$ & $n=17$ & $n=20$ & $n=23$ \\
\hline \hline maximum power transmission & 0.1074 & 0.1105 & 0.1198 & 0.1303 & 0.1331 & 0.1254 & 0.1276 & 0.1248 \\
\hline DSP algorithm & 0.0014 & 0.0024 & 0.0037 & 0.0058 & 0.0080 & 0.0103 & 0.0132 & 0.0161 \\
\hline
\end{tabular}

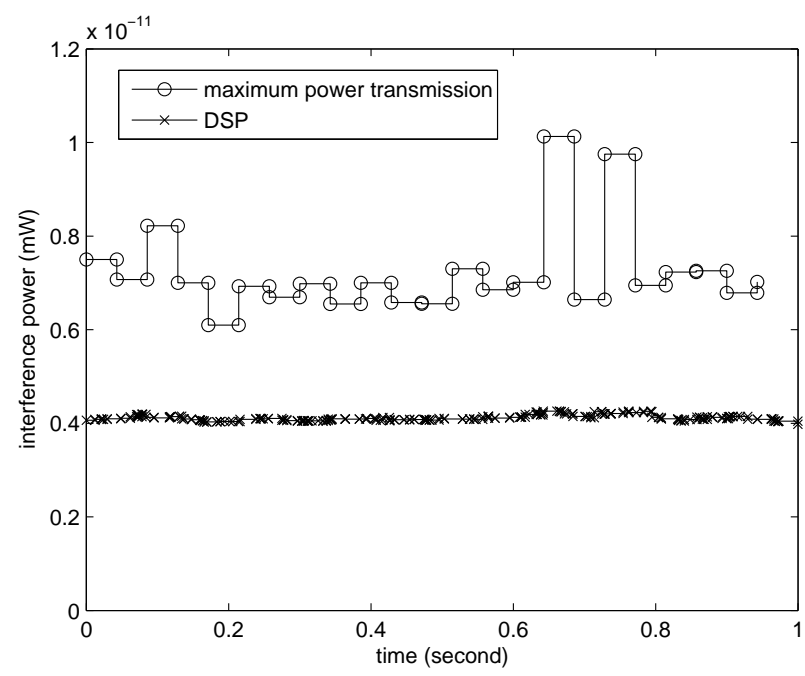

Fig. 7. The fluctuation of the interference power at the base station of the center cell $C(1)$ within one time frame.

very small, i.e., the interference power fluctuates very little. These results verify our constant interference assumption, which was the basis for the decomposition method proposed in this paper. The DSP algorithm has the effect of smoothing out the interference power received at the base stations. This observation further indicates that the scheduling order of the users in each cell is not important in the DSP algorithm. Our DSP algorithm can alleviate the combinatorial part in formulation (13), which is the most challenging part in solving the joint power control, rate control, and scheduling problem.

\section{Convergence}

When $\delta>0$, the DSP algorithm involves iterations between two alternative steps. The total power consumption is reduced in each iteration. The DSP algorithms terminates if the improvement in the current iteration is less than a percentage threshold. Figure 8 shows the number of iterations that the DSP algorithm needs for convergence. For each given number of links, we investigate 200 random networks and present both the maximum numbers and the average numbers of iterations of the DSP algorithm. We find that for all the simulated networks with different number of users per cell, the average number of iterations for DSP to converge is around 3. The maximum number of iterations of the DSP algorithm is no larger than 8. In Section IV-B, we show that the DSP algorithm is guaranteed to converge. Figure 8 further indicates that the DSP algorithm converges very fast.

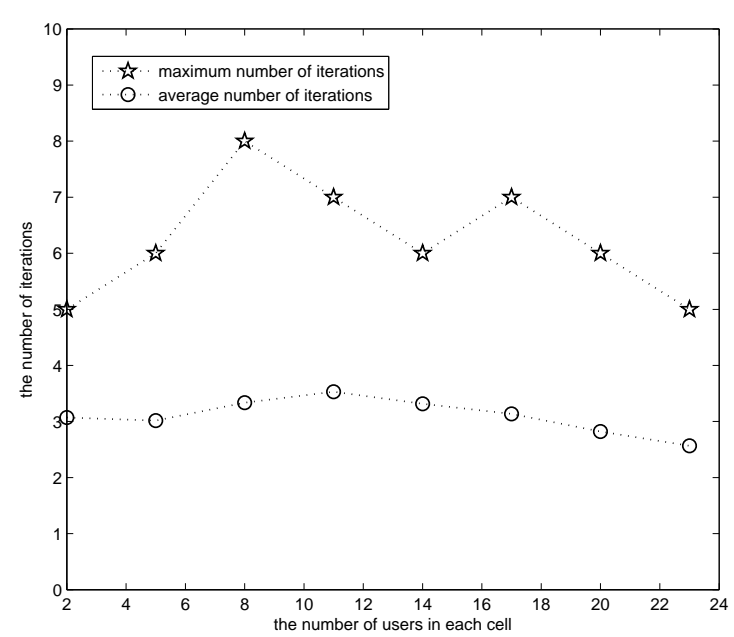

Fig. 8. The maximum and average numbers of iterations for the DSP algorithm $(\delta>0)$ to converge.

\section{FUTURE WORK}

In this section, we will discuss two possible future research directions: the first one is the energy conservation in cellular networks that support mobility; the second one is the energy conservation problem in the wireless networks that support non-real-time applications.

When users are moving, their channels are often fast timevarying. The power solutions of the DSP algorithm may not satisfy the users' target SINR requirements, since the channel gains may have been changed before the algorithm converges. One possible solution is to set an SINR margin to combat the negative impact of mobility [23], i.e., increase the target SINR by a certain amount. As a result, although the channel gains may have been changed, the users transmission rate requirements can still be satisfied if the SINR margin is sufficiently large. For example, a margin of $3 \mathrm{~dB}$ is reserved for up-link transmissions in mobile WiMAX assuming a frequency reuse factor of 3 [24]. It is clear that there is a tradeoff between the SINR margin and the energy efficiency: a small SINR margin may not guarantee the mobile users' QoS requirements; a large SINR margin may lead to unnecessary waste of energy consumptions. Furthermore, the optimization of the SINR margin is affected by several other factors, e.g., the moving speeds of the mobile users, the frequency reuse factor, and the frame length. The energy-efficient transmission in mobile multi-cell networks while providing QoS guarantees is an interesting topic for further study.

This paper focuses on the cellular networks that support 
real-time application sessions (video/ voice sessions). The extension to the dynamic systems that support non-real-time sessions (e.g., file transfers) is an interesting yet challenging topic. The real-time sessions have a special feature: the connection duration of a real-time session is independen$\mathrm{t}$ of the allocated network resource as long as its target rate requirement is satisfied; otherwise, the session may be dropped. However, non-real-time sessions are delay-tolerant. The holding time of a non-real-time session depends on the rate and power allocation policy. For example, allocating a lower transmission rate to a file transfer session will keep the corresponding user staying longer in the system. The stationary distribution of the number of users depends heavily on the rate and power control allocations. In addition, the QoS metric of non-real-time sessions is less stringent than the real-time sessions, and thus the system constraints are different. Therefore, the energy-conservation problem for nonreal-time sessions requires different formulation and solution techniques.

\section{CONCLUSION}

In this paper, we study the problem of energy conservation of terminals in a multi-cell TDMA network supporting bursty real-time sessions. The associated optimization problem involves joint scheduling, rate control, and power control.

We propose a method that decomposes the overall problem into two sub-problems: intra-cell energy optimization and inter-cell power control. This decomposition method is guaranteed to find a feasible solution, albeit not an optimal one. The decomposition is motivated and made simple by the following observations:

1) The original optimization problem is too complicated to solve directly online.

2) In cellular networks, the cells using the same frequency band are usually geographically separated by a distance. Interference is a strong function of distance when the distance is small, but a weak function of distance when the distance is large. Furthermore, after doing intra-cell averaged power minimization, the base station trades off energy consumption with transmission time. This will reduce the interference power generated by the cellboundary users. Thus, we could make the approximation that the interference is constant when we make intra-cell time fraction allocations to the users within a cell.

3 ) If the idle power is no less than the circuit power, or both are negligible, then there is a "decoupling property": the energy-optimal time allocations to individual users within each cell are independent of the inter-cell interference (under the assumption that the interference stays constant throughput a frame).

4) If the idle power is less than the circuit power, the subproblems are coupled. We then need to iteratively solve the two sub-problems until convergence.

\section{REFERENCES}

[1] "Nice talking to you ... mobile phone use passes milestone," in http://www.guardian.co.uk/technology/2009/mar/03/mobile-phones1, March 2009.
[2] J. Mogul, "Improving energy efficiency for networked applications," in Architectures for Networking and Communications Systems (ANCS) Keynote Speech, 2007.

[3] S. Cui, A. J. Goldsmith, and A. Bahai, "Energy-constrained modulation optimization," IEEE Trans. Wireless Commun., vol. 4, pp. 2349-2360, Sep. 2005.

[4] S. Cui, R. Madan, A. J. Goldsmith, and S. Lall, "Cross-layer energy and delay optimization in small-scale sensor networks," IEEE Trans. Wireless Commun., vol. 6, pp. 3688-3699, Oct. 2007.

[5] U. C. Kozat, I. Koutsopoulos, and L. Tassiulas, "Cross-layer design for power efficiency and QoS provisioning in multi-hop wireless networks," IEEE Trans. Wireless Commun., vol. 5, no. 11, pp. 3306-3315, Nov. 2006.

[6] B. Prabhakar, E. Uysal Biyikoglu, and A. El Gamal, "Energy-efficient transmission over a wireless link via lazy packet scheduling," in Proc. IEEE INFOCOM, vol. 1, 2001, pp. 386-394.

[7] G. J. Foschini and Z. Miljanic, "A simple distributed autonomous power control algorithm and its convergence," IEEE Trans. Veh. Technol., vol. 42, no. 4, pp. 641-646, Nov. 1993.

[8] R. Yates, "A framework for uplink power control in cellular radio systems," IEEE Jour. Select. Areas in Comm., vol. 13, pp. 1341-1347, 1995.

[9] M. Chiang, P. Hande, T. Lan, and C. W. Tan, Power Control in Wireless Cellular Networks, Foundation and Trends in Networking, July 2008.

[10] M. Johansson and L. Xiao, "Cross-layer optimization of wireless networks using nonlinear column generation," IEEE Trans. Wireless Commun., vol. 5, no. 2, pp. 435-445, Feb. 2006.

[11] A. Gjendemsjo, D. Gesbert, G. E. Oien, and S. G. Kiani, "Binary power control for sum rate maximization over multiple interfering links," IEEE Trans. Wireless Commun., vol. 7, pp. 3164-3173, Aug. 2008.

[12] S. Singh, N. B. Mehta, A. F. Molisch, and A. Mukopadhyay, "Momentmatched lognormal modeling of uplink interference with power control and cell selection," IEEE Trans. Wireless Commun., vol. 9, no. 3, pp. 932-938, Mar. 2010.

[13] H. Kim and G. de Veciana, "Leveraging dynamic spare capacity in wireless systems to conserve mobile terminals' energy," IEEE/ACM Trans. Networking., vol. 18, no. 3, pp. 802-815, June 2010.

[14] G. Miao, N. Himayat, G. Y. Li, and S. Talwar, "Distributed interferenceaware energy-efficient power optimization," IEEE Trans. Wireless Commun., vol. 10, no. 4, pp. 1323-1333, Apr. 2011.

[15] H. Kim, C.-B. Chae, G. de Veciana, and R. W. H. Jr., "A cross-layer approach to energy efficiency for adaptive MIMO systems exploiting spare capacity," IEEE Trans. Wireless Commun., vol. 8, no. 8, pp. 42644275, Aug. 2009

[16] WiMAX power amplifier ADL5570 and 5571, "http://www.analog. com/uploadedfiless/data \sheets/adl5570.pdf, http://www.analog.com/ uploadedfiless/data $\backslash$ sheets/adl5571.pdf”, Std. Analog Device, Sep. 2007.

[17] A. J. Goldsmith and P. P. Varaiya, "Capacity of fading channels with channel side information," IEEE Trans. Infomation Theory, vol. 43, no. 6, pp. 1986-1992, Nov. 1997.

[18] R. A. Horn and C. R. Johnson, Matrix Analysis. New York: Cambridge Univ. Press, 1991

[19] N. Bambos, C. Chen, and G. Pottie, "Channel access algorithms with active link protection for wireless communication networks with power control," IEEE/ACM Trans. Networking., vol. 8, no. 5, pp. 583-597, Oct. 2000 .

[20] D. Mitra, "An asynchronous distributed algorithm for power control in cellular radio systems," in Proc. 4th WINLAB Workshop, Rutgers University, New Brunswick, NJ, 1993.

[21] S. Boyd and L. Vandenberghe, Convex Optimization. Cambridge University Press, 2004.

[22] R. M. Corless, G. H. Gonnet, D. E. G. Hare, D. J. Jeffrey, and D. E. Knuth, "On the lambert W function," Advances in Computational Mathematics, vol. 5, pp. 329-359, 1996.

[23] C. W. Tan, D. P. Palomar, and M. Chiang, "Energy-robustness tradeoff in cellular network power control," IEEE/ACM Trans. Networking., vol. 17, no. 3, pp. 912-925, June. 2009.

[24] "Mobile WiMAX part I: A technical overview and performance evaluation," in http://www.wimaxforum.org/technology/downloads/Mobile_Wi MAX_Part1_Overview_and_Performance.pdf, Aug. 2006. 


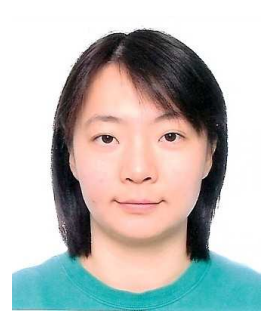

Liqun Fu is currently a postdoctoral fellow with the Institute of Network Coding at The Chinese University of Hong Kong. She received the B.S. degree (honors) in Electrical Engineering from Xiamen University (Xiamen, China) in 2003, the M.S. degree in Electrical Engineering from Tsinghua University (Beijing, China) in 2006, and the Ph.D. Degree in Information Engineering from The Chinese University of Hong Kong in 2010. She worked as a visiting research student in the Department of Electrical Engineering, Princeton University from June to November, 2009. Her current research interests are in the area of wireless communications and networking, with current focus on wireless greening, resource allocation, distributed protocol design, and physical-layer network coding

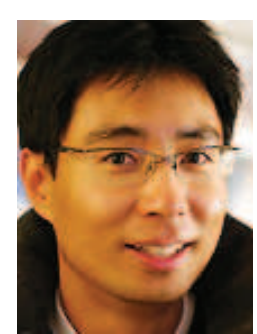

Hongseok Kim (S '06-M '10) is an Assistant Professor in the Department of Electronic Engineering at Sogang University, Seoul, Korea. He received his B.S. and M.S. degree in Electrical Engineering from Seoul National University in 1998 and 2000, respectively, and Ph.D. degree in Electrical and Computer Engineering at the University of Texas at Austin in 2009. He was a member of technical staff at Korea Telecom Network Laboratory, Daejeon, Korea from 2000 to 2005. He worked as a PostDoctoral Research Associate in the Department of Electrical Engineering at Princeton University, Princeton, NJ from 2009 to 2010 and worked as a Member of Technical Staff at Bell Labs, AlcatelLucent, Murray Hill, NJ from 2010 to 2011. His research interests are network resource allocation and optimization including cross layer design of wireless communication systems, MIMO and OFDMA, network economics, and Smart Grid. Dr. Kim is the recipient of Korea Government Overseas Scholarship in 2005-2008.

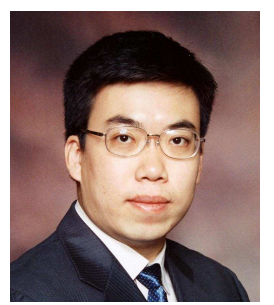

Jianwei Huang (S'01-M'06-SM'11) is an Assistant Professor in the Department of Information Engineering at the Chinese University of Hong Kong. $\mathrm{He}$ received B.S. in Electrical Engineering from Southeast University (Nanjing, Jiangsu, China) in 2000, M.S. and Ph.D. in Electrical and Computer Engineering from Northwestern University in 2003 and 2005, respectively. He worked as a Postdoc Research Associate in the Department of Electrical Engineering at Princeton University during 20052007. Dr. Huang currently leads the Network Communications and Economics Lab (ncel.ie.cuhk.edu.hk), with current research focuses on network economics, cognitive radio networks, and smart grid. $\mathrm{He}$ is the recipient of the IEEE Marconi Prize Paper Award in Wireless Communications in 2011, the IEEE GLOBECOM Best Paper Award in 2010, the IEEE ComSoc Asia-Pacific Outstanding Young Researcher Award in 2009, Asia-Pacific Conference on Communications Best Paper Award in 2009, and Walter P. Murphy Fellowship at Northwestern University in 2001.

Dr. Huang has served as Editor of IEEE Journal on Selected Areas in Communications (JSAC) - Cognitive Radio Series, Editor of IEEE Transactions on Wireless Communications, Guest Editor of IEEE JSAC special issues on "Economics of Communication Networks and Systems" and "Game Theory in Communication Systems", and Guest Editor of several other journals including (Wiley) Wireless Communications and Mobile Computing, Journal of Advances in Multimedia, and Journal of Communications.

Dr. Huang has served as Vice Chair of IEEE MMTC (Multimedia Communications Technical Committee), Director of IEEE MMTC E-letter, the TPC Co-Chair of IEEE WiOpt (International Symposium on Modeling and Optimization in Mobile, Ad Hoc, and Wireless Networks) 2012, the Publicity CoChair of IEEE Communications Theory Workshop 2012, the TPC Co-Chair of GameNets (the International Conference on Game Theory for Networks) 2009, the TPC Co-Chair of IEEE GlOBECOM Wireless Communications Symposium 2010, and the TPC Co-Chair of IWCMC (the International Wireless Communications and Mobile Computing) Mobile Computing Symposium 2010.

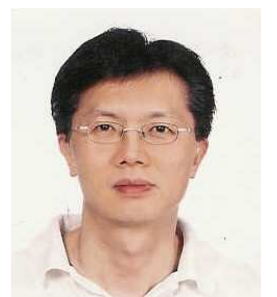

Soung Chang Liew received his S.B., S.M., E.E., and Ph.D. degrees from the Massachusetts Institute of Technology. From 1984 to 1988 , he was at the MIT Laboratory for Information and Decision Systems, where he investigated Fiber-Optic Communications Networks. From March 1988 to July 1993, he was at Bellcore (now Telcordia), New Jersey, where he engaged in Broadband Network Research. He has been Professor at the Department of Information Engineering, the Chinese University of Hong Kong, since 1993. He is Adjunct Professor at Southeast University, China.

Prof. Liew's current research interests include wireless networks, Internet protocols, multimedia communications, and packet switch design. Prof. Liew's research group won the best paper awards in IEEE MASS 2004 and IEEE WLN 2004. Separately, TCP Veno, a version of TCP to improve its performance over wireless networks proposed by Prof. Liew's research group, has been incorporated into a recent release of Linux OS. In addition, Prof. Liew initiated and built the first inter-university ATM network testbed in Hong Kong in 1993. More recently, Prof. Liew's research group pioneers the concept of Physical-layer Network Coding (PNC).

Besides academic activities, Prof. Liew is also active in the industry. He cofounded two technology start-ups in Internet Software and has been serving as consultant to many companies and industrial organizations. He is currently consultant for the Hong Kong Applied Science and Technology Research Institute (ASTRI), providing technical advice as well as helping to formulate R\&D directions and strategies in the areas of Wireless Internetworking, Applications, and Services.

Prof. Liew is the holder of seven U.S. patents and Fellow of IET and HKIE. He currently serves as Editor for IEEE Transactions on Wireless Communications and Ad Hoc and Sensor Wireless Networks. He is the recipient of the first Vice-Chancellor Exemplary Teaching Award at the Chinese University of Hong Kong. Publications of Prof. Liew can be found in www.ie.cuhk.edu.hk/soung.

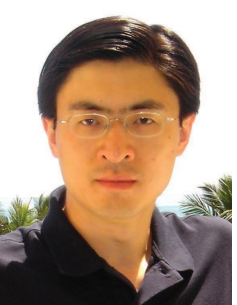

Mung Chiang is a Professor of Electrical Engineering at Princeton University, and currently serves as the Director of Graduate Studies in Electrical Engineering. He is also an affiliated faculty in Applied and Computational Mathematics, and in Computer Science. He received his B.S. (Hons.), M.S., and $\mathrm{Ph} . \mathrm{D}$. degrees from Stanford University in 1999, 2000, and 2003, respectively, and was an Assistant Professor 2003-2008 and a tenured Associate Professor 2008-2011 at Princeton University. $\mathrm{He}$ received the H. B. Wentz Junior Faculty Award from Princeton in 2005 and was a Hertz Foundation Fellow 1999-2003.

Chiang's research areas include the Internet, wireless networks, broadband access networks, content distribution networks, network economics, and online social networks. His research on networking received awards such as the IEEE Kiyo Tomiyasu Award in 2012, the U.S. Presidential Early Career Award for Scientists and Engineers in 2008, the Office of Naval Research Young Investigator Award in 2007, and the National Science Foundation CAREER Award in 2005. He received IEEE Globecom Best Paper Award three times. Chiang's inventions resulted in five issued patents, several technology transfers to commercial adoption, and the Technology Review TR35 Award in 2007. He founded the Princeton EDGE Lab in 2009.

Chiang was a co-chair of the 9th IEEE WiOpt Conference, the 38th CISS, and the 1st ACM S3 Workshop. He has been an associate or guest editor of IEEE Journal of Selected Areas in Communications, Transactions on Communication, Transactions on Information Theory, Transactions on Wireless Communications, IEEE/ACM Transactions on Networking, and Springer Journal of Optimization and Engineering. 